\title{
Comparative Regional and Subcellular Distributions of Histamine and Norepinephrine in the Hearts of Rats, Mice, Guinea Pigs, Rabbits, and Dogs
}

\author{
Stewart G. Harvey, Ph.D.
}

\section{Summary}

The patterns of distribution of histamine and norepinephrine among the 4 chambers of the heart of rats, guinea pigs, and rabbits and among 15 portions of the dog heart were quite similar, except for the coronary ring of the dog, which was disproportionately high in histamine. In whole mouse hearts the separate chambers were not assayed; in the whole heart, the contents of the 2 amines did not correlate. The subcellular distribution of histamine in the rat and guinea pig heart was different from that of norepinephrine. Histamine was mostly associated with mast cell-like granules. Toluidine blue-staining granules of 2 widely different densities were found.

\section{Additional Indexing Words:}

Histamine in heart Norepinephrine in heart Histamine relationship to norepinephrine Rat myocardial histamine Guinea pig myocardial histamine Rabbit myocardial histamine Mouse myocardial histamine Intracellular histamine in heart

D URING the course of an investigation on the histamine content of the thyroid gland, myocardial tissue was employed as one of several reference tissues to gauge the efficacy of histamine-depleting drugs. It was found that the histamine-releasing compound $48 / 80$ did not lower the histamine content of the heart. Consequently, an investigation into the content and nature of storage of histamine in the heart was undertaken. Pilot studies indicated not only that histamine and norepinephrine were distributed similarly but also at least part of the histamine could be depleted by reserpine. ${ }^{1)}$ Because of recent interest in possible histaminergic nerves, ${ }^{21-5)}$ the possibility was considered that myocardial histamine might be contained in sympathetic nerves, at least in part. For this reason, throughout this study both histamine and norepinephrine were studied in parallel. This report is an account of the similarities and dissimilarities of the myocardial distributions of histamine and norepinephrine.

From the Department of Pharmacology, University of Utah College of Medicine, Salt Lake City, Utah 84132, U.S.A.

Received for publication July 4, 1977. 


\section{Methods}

Animals: Only male rats were used. They were of the Sprague-Dawley strain. In pilot studies, weights ranged from 180 to $350 \mathrm{Gm}$, but all data reported herein were derived from rats weighing 180 to $220 \mathrm{Gm}$ at the outset of an experiment. These rats were litter mates. Mice were of a white Swiss strain; they weighed 18 to $22 \mathrm{Gm}$ at the outset of an experiment. Guinea pigs were of all colors; weights ranged from 300 to $450 \mathrm{Gm}$. White New Zeland rabbits were used; they weighed 3 to $4.5 \mathrm{Kg}$. No special breed of dog was used; weights ranged from 11.8 to $19.0 \mathrm{Kg}$.

Chemical assays: Histamine was determined by the method of Shore et al, ${ }^{6}$ ) except that citric acid, rather than hydrochloric acid, was used to terminate the reaction with o-phthaldialdehyde.") O-phthaldialdehyde was recrystallized from petroleum ether. All solvents were redistilled, and only the middle fraction was used.

Norepinephrine was determined by a modification of the trihydroxyindole method. In a $50 \mathrm{ml}$ polyethylene test tube (capped) $2 \mathrm{ml}$ of homogenate were added to $10 \mathrm{ml}$ of $1 \mathrm{M}$ tris buffer containing $0.0333 \mathrm{M}$ EDTA and $0.0243 \mathrm{M}$ $\mathrm{NaHSO}_{3}$, the $\mathrm{pH}$ of which solution was adjusted to 8.6 , and $200 \mathrm{mg}$ of acid-washed alumina (Woelm). The mixture was shaken in a horizontal direction at a rate of 1,700 1/4-inch strokes per min. After the supernatant was discared, the alumina residue was shaken with a wash of $2 \mathrm{ml}$ of tris buffer. The supernatant was again discarded, $2.5 \mathrm{ml}$ of $0.05 \mathrm{~N} \mathrm{HClO}_{4}$ was added, and the mixture was shaken for $10 \mathrm{~min}$. The alumina was packed by centrifugation, and the eluate was removed

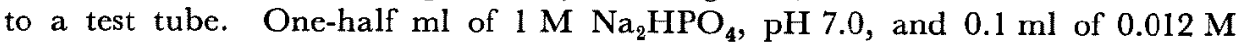
$\mathrm{K}_{3} \mathrm{FeC}_{6} \mathrm{~N}_{6}$ were added. After 1 minute, $1 \mathrm{ml}$ of $0.014 \mathrm{M}$ ascorbic acid in $10 \mathrm{~N}$ $\mathrm{NaOH}$ was added. The fluorometric reading was made immediately.

Separation of subcellular particles: A donor animal was anesthetized with sodium pentobarbital. The chest was opened and the heart quickly removed to a beaker containing Krebs-Henselheit solution at $4^{\circ} \mathrm{C}$. The heart was washed quickly to remove blood, and fat, adherent pieces of lung, trachea, etc were removed. The heart was opened and quickly blotted. Several methods of preparing the tissue prior to homogenization were tried, but, in all cases, the homogenization was performed by hand, rather than by motor-driven pestle. In one operation, the heart was cut into strips of approximately $100 \mathrm{mg}$; in a second, the heart was minced with scissors; in a third, the heart was pressed through a perforated lucite plate into a coarse mash. The strips, mince, or mash was placed into ten volumes of cold $0.25 \mathrm{M}$ or $1.20 \mathrm{M}$ sucrose, with or without buffer or other additives (see Results), and homogenized in an ice bath for various lengths of time and with varying amounts of pressure applied to the pestle. Glass tissue homogenizers fitted with teflon pestles were used. Homogenates were transferred to cold $50 \mathrm{ml}$ aged polyethylene centrifuge tubes. Centrifugations up to $20,000 \mathrm{~g}$ were performed in a Servall refrigerated centrifuge with a type SS-34 rotor. Supernatants that required sedimentation at a higher acceleration were transferred to cold lusteroid tubes and centrifuged in a Spinco preparative ultracentrifuge with a SW rotor. Sediments were carefully blotted to remove excess supernatant. Supernatants and sediments were stored in an ice bath if they could not be worked with immediately, but at no time was such storage longer than $5 \mathrm{~min}$. The sediment pellet was transferred to a glass 
tissue homogenizer containing $5 \mathrm{ml}$ of $0.4 \mathrm{~N} \mathrm{HClO}_{4}$, after which the procedure for assays of histamine and norepinephrine were performed as noted above. For densitygradient separations, the gradients were prepared either by placing $1 \mathrm{ml}$ of the most dense solution in a lusteroid tube and carefully layering the $1 \mathrm{ml}$ of next dense solution above it, continuing the process until all layers were in place, or by freezing each layer after it was added; such a frozen, layered medium was melted just prior to use. The freezing method did not appear to cause problems of solvent-solute separation, the interfaces were extremely sharp, and results were the same as those in which the media had not been frozen. After centrifugation, each layer was removed by means of a syringe fitted with a long 22 gauge needle from which the bevel had been removed.

Succinic dehydrogenase activity: Succinic dehydrogenase activity was determined by the method of Tam and Wilson.8)

\section{RESULTS}

Regional distribution of myocardial histamine: In the rat heart, the 4 chambers were assayed for histamine and norepinephrine. The histamine content was usually 2-3 times that of norepinephrine. Three principal patterns of distribution were observed (Fig. 1). In a group of litter mates more than $70 \%$ would show the same pattern of distribution. The pattern shown in the left-hand panel was the predominant one; 5 of 7 groups of animals showed this pattern. Most important is the fact that, irrespective of the pat-

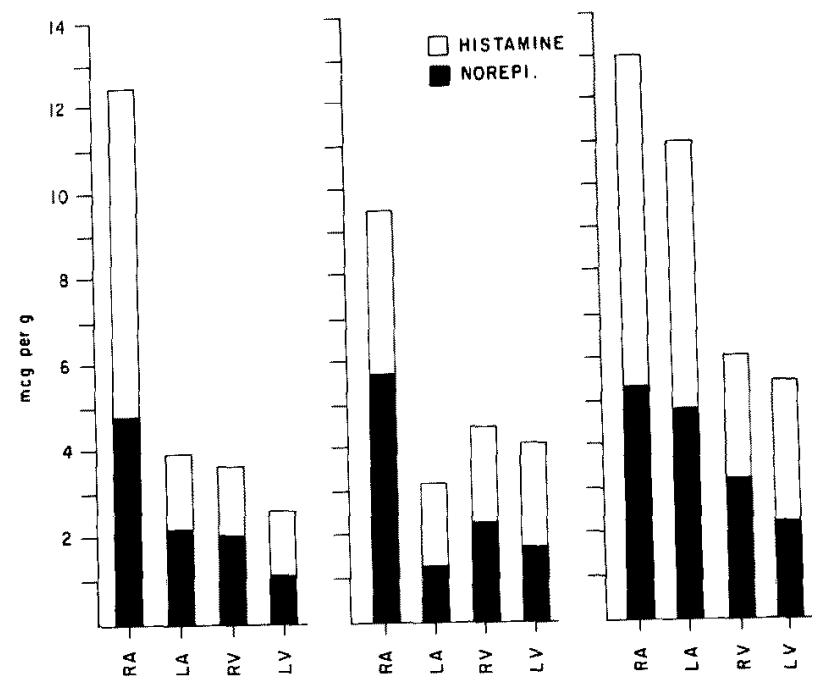

Fig. 1. Patterns of distribution of histamine and norepinephrine in the rat heart. $R A=$ right atrium; $L A=$ left atrium; $R V=$ right ventricle; $L V=$ left ventricle. Patterns vary, but are alike in most litter mates. The 3 patterns shown are the most common. 
tern, the pattern was similar for histamine and norepinephrine in more than $80 \%$ of the hearts. The chamber with the highest content of either amine was always the right atrium. Fig. 2 is a scatter diagram that shows the relationship of histamine concentration to norepinephrine concentration in 100 samples from 25 rats. In 4 other series of observations (not shown) the findings were nearly identical. For the data shown in Fig. 2, the correlation coefficient was 0.740 . In the guinea pig heart, the patterns of distribution were

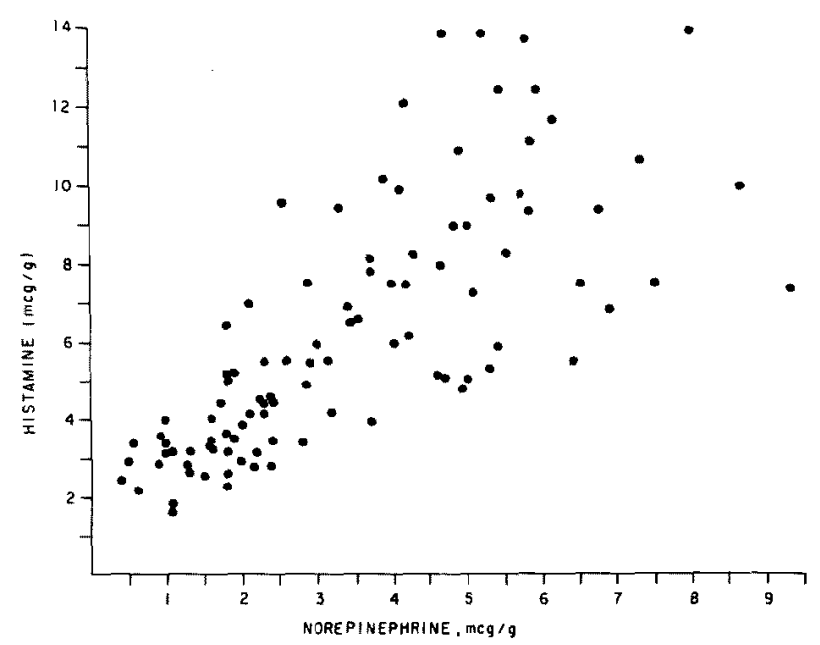

Fig. 2. The relationship of histamine concentration to norepinephrine concentration in the rat heart.

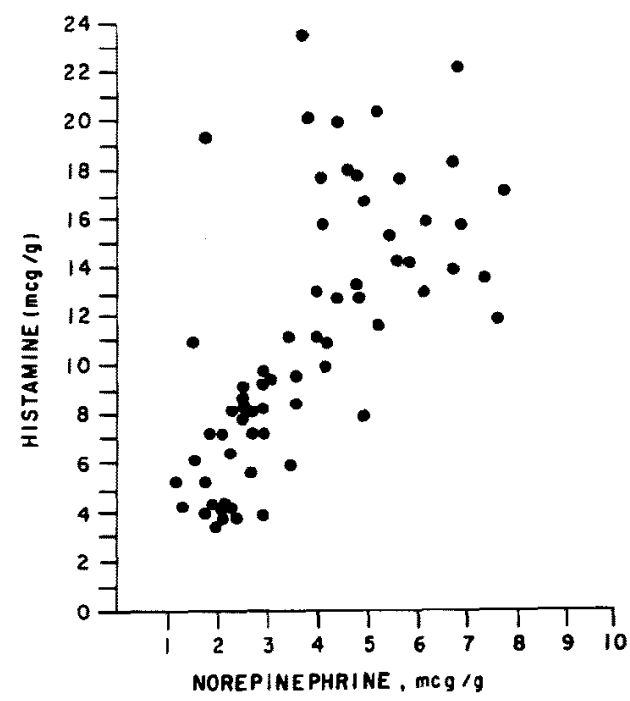

Fig. 3. The relationship of histamine concentration to norepinephrine concentration in the guinea pig heart. 


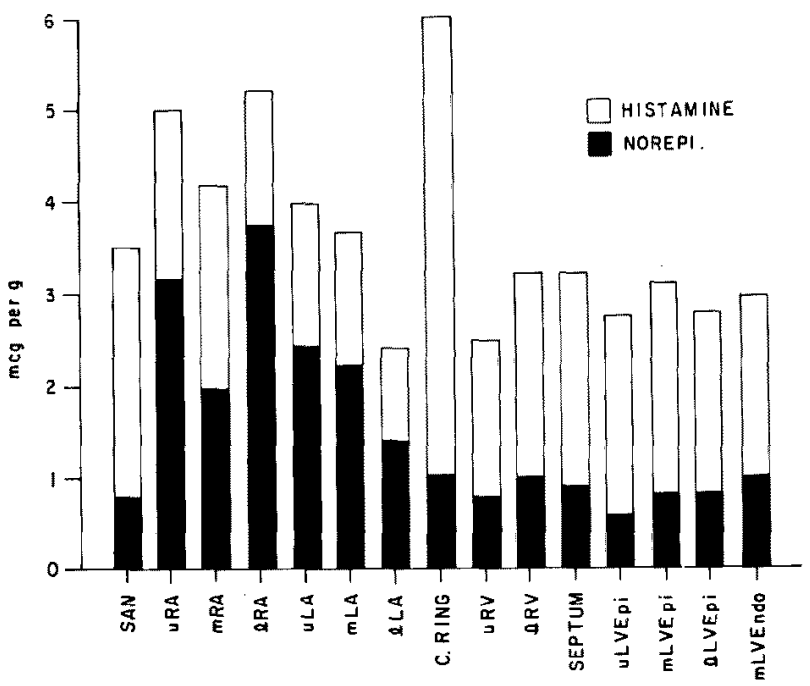

Fig. 4. Distributions of histamine and norepinephrine in the dog heart. $\mathrm{SAN}=$ region of sinoatrial node; $\mathrm{uRA}=$ upper right auricle; $\mathrm{mRA}=$ base of right auricle; $1 R A=$ wall of main chamber of right atrium; uLA, mLA, ILA are analogous to the designations for the right atrium, except that $\mathrm{L}=\mathrm{left}$; G. $\mathrm{RING}=$ coronary ring; $\mathrm{uRV}=$ upper portion of wall of right ventricle; $\mathrm{IRV}=$ lower portion of wall of right ventricle; SEPTUM = interventricular septum; uLVEpi=epicardial surface of upper portion of wall of left ventricle; $m$ \& $1 \mathrm{LVEpi}=$ similarly the middle and lower portions; mLVEndo= endocardial surface of middle portion of wall of left ventricle.

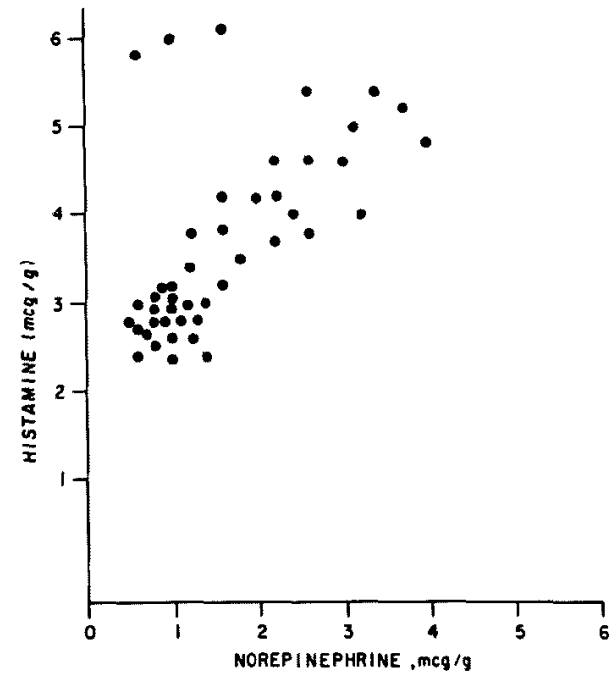

Fig. 5. The relationship of histamine concentration to norepinephrine concentration in the dog heart. The 3 points in the upper left represent the coronary ring. (See Fig. 4) 
similar to those of the rat heart, but the close parallelism of the histamine pattern with the norepinephrine pattern was not as frequent. A scatter diagram of histamine concentration as a function of norepinephrine concentration is shown in Fig. 3. The correlation coefficient was 0.613 . In each dog heart, 15 samples were assayed. Fig. 4 shows the distribution in one heart. In general, the histamine content was considerably higher than the norepinephrine content, but the profiles of the 2 amines were similar. The principal exception is the fibrous coronary ring, which contained more histamine than any other portion. The patterns were similar in all 3 dogs. A scattergam or relationship between histamine content and norepinephrine content is shown in Fig. 5. Except for the 3 points at the top of the graph, which represent the coronary rings, the correlation was better than with the other 3 species examined. The correlation coefficient was 0.603 when the data from the coronary rings were included and 0.869 when they were excluded. The hearts of 4 rabbits were studied. The patterns of distribution of histamine in each heart was the same as that of norepinephrine; the data are now shown. Fifty whole mouse hearts were assayed. There was no correlation between the contents of histamine and norepinephrine. The histamine content of mouse hearts was found to be $0.306 \pm .051 \mathrm{mcg} / \mathrm{Gm}$.

Subcellular distribution of myocardial histamine: The subcellular distributions of histamine and norepinephrine in rat and guinea pig hearts were investigated by both differential centrifugation and density-gradient techniques. Several media for homogenization and suspension and various accelerations and durations of centrifugation were investigated. Accelerations of $480,60,1,000,3,000,8,000,8,700,10,000,18,800,20,000$, and $105,000 \mathrm{~g}$ for various lengths of time were tested for serviceability in differential centrifugation. In $0.25 \mathrm{M}$ sucrose the optimum dispersion with the minimum release of the amines was found when $1,000 \mathrm{~g}$ for $5 \mathrm{~min}, 500 \mathrm{~g}$ for $15 \mathrm{~min}$, $20,000 \mathrm{~g}$ for $15 \mathrm{~min}, 105,000 \mathrm{~g}$ for $5 \mathrm{~min}$, and $105,000 \mathrm{~g}$ for $60 \mathrm{~min}$ were used in succession. Recoveries of the amines in the various fractions were the same whether the sucrose solution contained $0.001 \mathrm{M} \mathrm{MgCl}_{2}$ and $0.005 \mathrm{M}$ $\mathrm{K}_{2} \mathrm{HPO}_{4}$, buffered to $\mathrm{pH} 7.4$, or $0.05 \mathrm{M}$ tris-EDTA at $\mathrm{pH} 7.4$. Furthermore, the addition of $0.02 \mathrm{M}$ ATP did not affect the distribution. The distribution in tris-EDTA is shown in Table I. Each value is the mean result of 8 experiments in the rat and 4 in the guinea pig. In these experiments, the heart samples were pressed through a well-aged, perforated lucite plate before being homogenized by 7 hand strokes of the pestle. The sum of the recoveries of the amines in the several fractions ranged from 95.8 to $98.1 \%$ of the total content of unfractionated homogenates. The major quantity of either amine was found in the supernatant, but that of histamine was nearly 
Table I. Histamine and Norepinephrine in Subcellular Particles from Hearts of Rats and Guinea Pigs

\begin{tabular}{l|cc|cc}
\hline \multirow{2}{*}{ Fraction } & \multicolumn{2}{|c|}{ Rats } & \multicolumn{2}{c}{ Guinea Pigs } \\
\cline { 2 - 4 } & $\begin{array}{c}\text { Histaminet } \\
(\%)\end{array}$ & $\begin{array}{c}\text { Norepi* } \\
(\%)\end{array}$ & $\begin{array}{c}\text { Histamine } \\
(\%)\end{array}$ & $\begin{array}{c}\text { Norepi } \\
(\%)\end{array}$ \\
\hline $1,000 \mathrm{~g} \times 5 \mathrm{~min}$ & 9.5 & 12.8 & 17.8 & 9.7 \\
$5,000 \mathrm{~g} \times 15 \mathrm{~min}$ & 21.6 & 1.6 & 29.4 & 2.4 \\
$20,000 \mathrm{~g} \times 15 \mathrm{~min}$ & 3.2 & 6.3 & 2.2 & 2.8 \\
$105,000 \mathrm{~g} \times 5 \mathrm{~min}$ & 0.8 & 2.1 & 0 & 3.9 \\
$105,000 \mathrm{~g} \times 15 \mathrm{~min}$ & 0 & 38.2 & 0.5 & 39.7 \\
Supernatant & 64.9 & 40.0 & 50.1 & 42.3 \\
* Norepinephrine & & & & \\
+ Percent of the total amine content determined by summing the contents of the several fractions.
\end{tabular}

$25 \%$ higher than that of norepinephrine. In the particulate fractions the greatest amount of histamine was found in the 5,000 g-sediment while that of norepinephrine was in the $105,000 \mathrm{~g} \times 60 \mathrm{~min}$-sediment. Fewer strokes of the pestle increased the quantity of either amine in the $1,000 \mathrm{~g}$-sediment at the expense of the major amine-containing sediment. More vigorous homogenization increased the quantity of either amine in the supernatant at the expense of both its major sediment and the $1,000 \mathrm{~g}$-sediment. Toluidine blue was added to the homogenate prior to centrifugation to determine its distribution among the sediments. The 1,000 g-sediment stained moderately blue; microscopic inspection of this sediment revealed some intact mast cells, but the stain was also fixed in the interstices and in unidentifiable fragments. The 5,000 g-sediment stained intensely blue; however, this sediment contained lightly stained particles among the darkly stained ones. The dark particles were spheroid in shape. The $20,000 \mathrm{~g}$-sediment contained scattered darkly stained particles. The $105,000 \mathrm{~g} \times 60 \mathrm{~min}$-sediment was colorless, but the 5 min-sediment contained a few densely stained particles. When Janus B green was used as a stain, the $20,000 \mathrm{~g}$-sediment took a green color; there was also a purple layer at the top. This sediment contained variously shaped particles, of which approximately one-fourth had the classical oblate shape of mitochondria. This sediment demonstrated considerable succinic dehydrogenase activity. The 5,000 g-sediment consisted of 2 layers, a lower one which was colorless and an upper one which was light green. These layers could be separated at $3,000 \mathrm{~g} \times 25 \mathrm{~min}$. The $3,000 \mathrm{~g}$-sediment was colorless, was stained purple by methylene blue and did not manifest succinic dehydrogenase activity. The 5,000 g-residual fraction contained a few fragments resembling those in the $20,000 \mathrm{~g}$-sediment, and it had a slight succinic dehydrogenase activity. The $105,000 \mathrm{~g}$-sediments stained only very lightly 
Table II. Density Gradient Separation of Histamine- and NorepinephrineContaining Subcellular Particles from Hearts of Rats

\begin{tabular}{c|c|c}
\hline Sucrose Concentration & Histamine (\%) & Noradrenaline (\%) \\
\hline $0.25 \mathrm{M}$ & 37.7 & 32.9 \\
$0.35 \mathrm{M}$ & 20.0 & 27.1 \\
$0.50 \mathrm{M}$ & 8.2 & 21.3 \\
$0.75 \mathrm{M}$ & 25.6 & 9.4 \\
$1.00 \mathrm{M}$ & 1.1 & 5.0 \\
Sediment & 6.4 & 4.3
\end{tabular}

* Percent of total amine content determined by summing the contents of the several fractions.

with Janus B green and had no succinic dehydrogenase activity. The 1,000 g-sediment showed 2 layers with Janus B green, only the top of which was green. The lower layer could be separated by centrifuging for $5 \mathrm{~min}$ at 480 g. Histamine was found in both fractions, but norepinephrine was found only in the upper fraction. Both fractions had succinic dehydrogenase activity.

The distributions of histamine and norepinephrine were also different when separated by the density gradient method (Table II). Considerable quantities of both were found in the $0.25 \mathrm{M}$ fraction, which corresponded to the supernatant fraction obtained by differential centrifugation. The largest amount of particle-bound histamine was in $0.75 \mathrm{M}$ sucrose, whereas that for norepinephrine was in $0.50 \mathrm{M}$ sucrose. When the $0.75 \mathrm{M}$ fraction was diluted to $0.25 \mathrm{M}$, the histamine could be sedimented with $5000 \mathrm{~g} \times 15 \mathrm{~min}$. The sediment stained intensely with toluidine blue. A second sediment could be obtained with $20,000 \mathrm{~g}$; this sediment contained microscopically identifiable mitochondria, stained intensely with Janus $B$ green, and had high succinic dehydrogenase activity. Of the histamine in the $0.35 \mathrm{M}$ fraction, none sedimented at $5,000 \mathrm{~g}$ after dilution, some sedimented at $20,000 \mathrm{~g}$, some sedimented at $105,000 \mathrm{~g}$, and approximately half remained in the supernatant; the 5,000 g-sediment stained lightly with toluidine blue. When the $0.5 \mathrm{M}$ fraction was diluted $0.25 \mathrm{M}$ and differentially centrifuged, the norepinephrine sedimented at $105,000 \mathrm{~g}$. The $1.00 \mathrm{M}$ sucrose fraction stained moderately with Janus B green and showed a marked succinic dehydrogenase activity. The sediment from this layer contained only small amounts of either amine.

Nuclei, large myocardial cell fragments, and other debris were found mostly in the sediment below the $1.00 \mathrm{M}$ layer. 


\section{Discussion}

The histamine content of rat heart has been reported to range from $1.3-2.3 \mathrm{mcg} / \mathrm{Gm}^{9)}$ to $4-6 \mathrm{mcg} / \mathrm{Gm}^{6}{ }^{6,10), 11)}$ Whole hearts were not assayed in the present work, so that it is not possible accurately to compare the present data with those of the sources cited. However it would appear from the present data that the content of the whole heart would generally be intermediate to the extremes cited. There was considerable variation in the values determined at different times. All rats were obtained from the same supplier and presumably were all maintained on the same dict. The variations were probably not due to differences in age, since all the data reported were obtained from rats of approximately the same weight. It was noted from unreported experiments that the histamine contents were higher in old than in young rats. The histamine content of the dog heart reported in the present study is consistent with that reported in the literature, but the content in guinea pig heart is higher. ${ }^{12}$ )

One of the more interesting aspects of the present findings is the striking parallelism in the distributions of histamine and norepinephrine in the heart. The correlations depicted in Figs. 2, 3, and 5, however, are only obtainable when the samples are derived from different portions of the heart. If only the valuesof a single chamber are plotted, no correlation exists. In other words, the correlations result from differences in chamber-to-chamber distribution of each amine. This is why no correlation was evident in the mouse heart, since only whole hearts were assayed. Nevertheless, the question of why histamine and norepinephrine are distributed in parallel among the various portions of the heart except, for the coronary ring of the dog, is most intriguing. The coronary ring, of course, is fibrous and would be expected to contain mast cells in greater abundance than the muscular portions of the heart.

By differential centrifugation, the subcellular distribution of histamine is what would be expected for mast cells, namely, that the principal particulate non-cellular fraction does not sediment at $1,000 \mathrm{~g}$ but does at $5,000 \mathrm{~g}$. To avoid mitochondria, Thon and Uvnäs ${ }^{13)}$ isolated mast cell granules with $27,000 \mathrm{~g}$ rather than $5,000 \mathrm{~g}$. In the present work at $5,000 \mathrm{~g}$, contamination by mitochondria appeared to be slight. That the 5,000 g-sediment stained intensely with toludine blue suggests that this sediment contained a large number of mast cell granules. The histamine in the $1,000 \mathrm{~g}$-sediment was probably mostly contained in unbroken cells.

Whether the large amount of histamine in the supernatants originated from disrupted granules or represented a cytosol pool of histamine was not determined. Mast cell granules appear to be very stable in isotonic sucrose 
solution, ${ }^{13)}$ so that disruption of granules would be expected to be slight. In the brain, in which organ the histamine is generally considered to be nonmast cell in nature, by differential centrifugation a large proportion of the histamine is found in the supernatant fraction, although it is also found in the mitochondrial and " nuclear" fractions. ${ }^{14)}$ The presence or absence of buffer, magnesium, or ATP did not seem to affect the proportion of histamine or of epinephrine in the supernatant.

By density gradient separation, the subcellular distribution is harder to to interpret. The histamine found in the $0.25 \mathrm{M}$-fraction corresponds to the supernatant from differential centrifugation. The finding that the particlebound histamine was in 2 fractions was surprising. That the histamine in the $0.36 \mathrm{M}$-fraction was not all contaminate from the layer above was shown by the fact that much of the histamine could be sedimented after dilution of the sucrose to $0.25 \mathrm{M}$. This fraction appeared to correspond only in part to the $5,000 \mathrm{~g}$-fraction by differential centrifugation. The fact that some histamine sedimented at $20,000 \mathrm{~g}$ and some at $105,000 \mathrm{~g}$ is of interest. Although the $0.73 \mathrm{M}$-fraction appeared to contain mitochondria, the fact that it stained intensely with toluidine blue indicates that mast cell granules were in this fraction. The appearance of $5,000 \mathrm{~g}$-sedimentable histamine in both the $0.35 \mathrm{M}$ and $0.75 \mathrm{M}$ fractions would indicate that there are mast cell-like granules of 2 considerably different densities. That these 2 histaminecontaining fractions are less dense than most of the mitochondria does not agree with the order in which they sediment by differential centrifugation, since the granules were smaller than mitochondria. A further discrepancy between the 2 methods is that by density-gradient separation there was a lesser quantity of either amine, especially norepinephrine, in the sediment than in the $1,000 \mathrm{~g}$-sediment by differential centrifugation. In the present work, the mitochondria were found in the $1.00 \mathrm{M}$-fraction, whereas in the work of Potter and Axelrod ${ }^{15}$ they were found principally at a density of about $0.8 \mathrm{M}$. However, in their work the duration of centrifugation was only $30 \mathrm{~min}$. Furthermore, an appreciable proportion of the norepinephrine was found in a lighter density fraction $(0.35 \mathrm{M})$ than found by other workers; ${ }^{16}$ ) however, in the present study, a considerable amount was found in the $0.5 \mathrm{M}$-fraction, in agreement with other reports. Consequently, the present data must be accepted cautiously. In the brain, by density gradient separation, the distribution of particle-bound histamine is also bimodal, ${ }^{14)}$ with peaks that correspond to concentrations of about 0.75 and $1.0 \mathrm{M}$ sucrose, whereas the 2 modes in this work are more widely separated.

Even though the fact that the gross distribution of histamine is similar to that of norepinephrine may suggest that myocardial histamine is in some 
way associated with adrenergic nerves, the differences in the subcellular disstribution are sufficient to indicate that the 2 amines are not stored in the same vesicle. The staining properties of the histamine-containing particles are like those of mast cell granules, even though the sedimentary properties seem to be different.

\section{Acknowledgements}

This study was supported by a grant-in-aid from the Utah Heart Association. The author is indebted to Neil H. Price and Richard B. Pannell for excellent technical assistance in this study.

\section{REFERENCES}

1. Harvey SG: Some characteristics of histamine storage in the heart. Fed Proc 30: 1696, 1971

2. Beck L: Histamine as the potential mediator of active reflex dilation. Fed Proc 24: 1298, 1965

3. Ehinger B: Uptake of histamine metabolites into sympathetic nonadrenergic neurons. Acta Physiol Scand 90: 218, 1974

4. Garbarg M, Barbin G, Feger J, Schwarsz J-G: Histaminergic pathway in rat brain evidenced by lesions of the medial forebrain bundle. Science 186: 833, 1974

5. Ryan MJ, Brody MJ: Distribution of histamine in the canine autonomic nervous system. J Pharmacol Exp Ther 174: 123, 1970

6. Shore PA, Burkhalter A, Cohn VH Jr: A method for the fluorometric assay of histamine in tissues. J Pharmacol Exp Ther 127: 182, 1959

7. Anton AH, Sayre DF: A modified fluorometric procedure for tissue histamine and its distribution in various animals. J Pharmacol Exp Ther $166: 285,1969$

8. Tam RK, Wilson PW: Respiratory enzyme systems in symbiotic nitrogen fixation, III. The dehydrogenase systems of Rhizobium trifolii and Rhizobium leguminosarum. J Bact 41 : 529,1941

9. Michaelson IA, Smithson HR: Effect of NSD-1055 on tissue levels of histidine and spermidine in rat. Proc Soc Exp Biol Med 136: 660, 1971

10. Feldberg, Talesnik J: Reduction of tissue histamine by compound $48 / 80$, J Physiol (Lond) 120: 3521953

11. Johnson HL: Nonmast-cell histamine kinetics. III. Uptake, metabolism and decline of $\mathbf{H}^{3}$ histamine in the female rat and effects of endogenous histamine release. J Pharmacol Exp Ther $171: 88,1970$

12. Mannaioni PF: Physiology and pharmacology of cardiac histamine. Arch internat Pharmacodyn Ther 196 (suppl): 64, 1972

13. Thon IL, Uvnäs B: Mode of storage of histamine in mast cells. Acta Physiol Scand 67; 455,1966

14. Kuhar MJ, Taylor KM, Snyder SH: The subcellular localization of histamine and histamine methyltransferase in rat brain. J Neurochem 18: 1515, 1971

15. Potter LT, Axelrod J: Subcellular localization of catecholamines in tissues of the rat. J Pharmacol Exp Ther 142: 291, 1963

16. Roth RH, Stjärne L, Bloom FE, Giarman NJ: Light and heavy noradrenaline storage particles in the rat heart and in bovine splenic nerve. J Pharmacol Exp Ther 162: 203, 1968 